\section{Camera Calibration from Images of Spheres}

\author{
Hui Zhang, Student Member, IEEE, \\ Kwan-Yee K. Wong, Member, IEEE, and \\ Guoqiang Zhang, Student Member, IEEE
}

\begin{abstract}
This paper introduces a novel approach for solving the problem of camera calibration from spheres. By exploiting the relationship between the dual images of spheres and the dual image of the absolute conic (IAC), it is shown that the common pole and polar with regard to the conic images of two spheres are also the pole and polar with regard to the IAC. This provides two constraints for estimating the IAC and, hence, allows a camera to be calibrated from an image of at least three spheres. Experimental results show the feasibility of the proposed approach.
\end{abstract}

Index Terms-Calibration, sphere, silhouette, surface of revolution (SOR).

\section{INTRODUCTION}

WITH the development of modern vision applications, multiview vision systems have become more and more cost effective. The traditional way of calibrating such a large number of cameras requires the use of some precisely made calibration patterns. However, such an approach is often tedious and cumbersome since points on the calibration pattern may not be simultaneously visible in all views. Besides, existing methods generally require knowledge of the metric structure [1], [2] of the calibration pattern. This will involve the design and use of some highly accurate tailormade calibration patterns, which are often difficult and expensive to manufacture. To overcome these difficulties, it is desirable to have some common simple object, such as surfaces of revolution (SOR) [3] or spheres [4], [5], [6], to replace the calibration patterns.

This paper uses spheres as a calibration object. The silhouettes of a sphere can be extracted reliably from images and this facilitates precise camera calibration. Besides, as long as the sphere is placed in the common field of view of the cameras, its occluding contours are always visible from any position and their images can be recovered even under partial occlusion. Spheres hence can be used to accurately calibrate multiple cameras mounted at arbitrary locations simultaneously. Spheres were first used in [7] to compute the aspect ratio of the two image axes. Daucher et. al. [8] later introduced a multistep nonlinear approach to estimate four camera parameters using spheres. However, error seriously accumulated in the separated steps. More recently, Teramoto and Xu [4] related the absolute conic with the images of spheres and calibrated the camera by minimizing the reprojection errors nonlinearly. Nevertheless, the final results of their method depend greatly on the initialization. Agrawal and Davis [9] derived similar constraints as in [4] in the dual space. Their method first estimates the imaged sphere centers and the remaining parameters are then solved by minimizing some algebraic errors with nonlinear semidefinite programming. However, there could be no solution when the noise is large. Further, apart from the five camera intrinsic parameters, 12 other parameters have to be estimated, which might ruin the precision of the results.

This paper proposes an approach to solve the above problems by exploiting the relationship between the dual images of spheres and the dual image of the absolute conic. It is shown that a conic homography can be derived from the conic matrices of the imaged

- The authors are with the Department of Computer Science, The University of Hong Kong, Pokfulam Road, Hong Kong.

E-mail: \{hzhang, kykwong, gqzhang\}@cs.hku.hk.

Manuscript received 13 July 2005; revised 10 Feb. 2006; accepted 5 July 2006; published online 15 Jan. 2007.

Recommended for acceptance by K. Daniilidis.

For information on obtaining reprints of this article, please send e-mail to: tpami@computer.org, and reference IEEECS Log Number TPAMI-0369-0705. spheres and the axis and vertex of such a homography are the pole and polar with regard to the image of the absolute conic. Some preliminary results have been published in [10]. Inspired by [3], the polar thus obtained can also be regarded as the imaged revolution axis of a surface of revolution (SOR) formed by the two spheres. The pole then corresponds to the vanishing point of the normal direction of the plane formed by the camera center and the two sphere centers. This again gives the pole-polar relationship with regard to the IAC. The orthogonal constraints [11] can then be used to estimate the IAC from the pole and polar obtained and calibrate the camera. Experiments show that this approach has good precision and can be used directly in practical reconstruction.

This paper is organized as follows: Section 2 presents the theory for camera calibration from the imaged absolute conic. Section 3 relates the dual image of a sphere to that of the absolute conic (IAC). Section 4 introduces our novel linear approach for camera calibration from spheres. Section 5 shows the results of synthetic and real experiments. Section 6 discusses the degenerate cases and Section 7 gives the conclusions.

\section{Calibration with the Absolute Conic}

The absolute conic was first introduced by Faugeras et al. [12] for camera self-calibration. It is a point conic on the plane at infinity that is invariant to similarity transformation. Let the camera calibration matrix be

$$
\mathbf{K}=\left[\begin{array}{ccc}
\alpha f & s & u_{0} \\
0 & f & v_{0} \\
0 & 0 & 1
\end{array}\right]
$$

where $f$ is the focal length, $\alpha$ is the aspect ratio, $\left(u_{0}, v_{0}\right)$ is the principal point, and $s$ is the skew. The image of the absolute conic (IAC) is then given by [12]

$$
\omega=\mathbf{K}^{-\mathbf{T}} \mathbf{K}^{-1}=\left[\begin{array}{lll}
\omega_{11} & \omega_{12} & \omega_{13} \\
\omega_{12} & \omega_{22} & \omega_{23} \\
\omega_{13} & \omega_{23} & \omega_{33}
\end{array}\right] .
$$

Note that $\omega$ is a symmetric matrix defined up to an unknown scale, hence it has five degrees of freedom.

The images of the absolute conic and its dual (DIAC) $\omega^{*}=\mathbf{K K}^{\mathbf{T}}$ [11] are the 2D projections of the 3D invariant absolute conic (AC) and the dual of the absolute conic (DAC), respectively. The IAC and DIAC are imaginary point and line conics from which the camera calibration matrix $\mathbf{K}$ can be easily obtained by Cholesky decomposition [13]. A camera with $s=0$ is called a zero skew camera and this results in $\omega_{12}=0$. When both $s=0$ and $\alpha=1$, the camera is called a natural camera and this results in $\omega_{12}=0$ and $\omega_{11}=\omega_{22}$. The IAC $\omega$ can be estimated using the orthogonal constraints [11], which states that the vanishing point $\mathbf{v}$ of the normal direction of a plane and the vanishing line 1 of the plane must satisfy the pole-polar relationship with regard to $\omega$, i.e.,

$$
\mathbf{l}=\omega \mathbf{v}
$$

This provides two independent constraints on the elements of $\omega$. Hence, to fully calibrate a camera, at least three such conjugate pairs are needed; for a zero skew or a natural camera, at least two pairs are needed.

\section{The Apparent Contour of a Sphere and ItS DUAL}

This section relates the IAC with the image of spheres. Consider first a particular case where a camera $\mathbf{P}=\mathbf{K}\left[\mathbf{I}_{3} \mid \mathbf{0}\right]$ is viewing a sphere centered at the $Z$-axis (see Fig. 1a). The limb points $\mathbf{X}=$ $\left[r \cos \theta r \sin \theta Z_{0} 1\right]^{\mathbf{T}}$ of the sphere always form a circle $\mathbf{C}_{3}$ with radius $r$ on the plane $Z=Z_{0}$. The image points $\hat{\mathbf{x}}$ (see Fig. 1b) of $\mathbf{X}$ under $\mathbf{P}$ can be defined as 


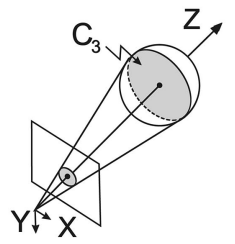

(a) (b)

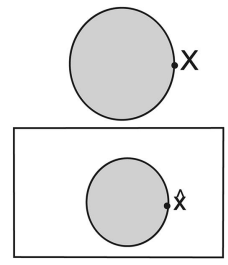

Fig. 1. (a) A sphere being viewed by a camera. (b) The limb points of the sphere are projected to a circle in the image.

$$
\hat{\mathbf{x}}=\mathbf{K}\left[\mathbf{I}_{3} \mid 0\right]\left[\begin{array}{c}
r \cos \theta \\
r \sin \theta \\
Z_{0} \\
1
\end{array}\right]=r \mathbf{K}\left[\begin{array}{ccc}
1 & 0 & 0 \\
0 & 1 & 0 \\
0 & 0 & Z_{0} / r
\end{array}\right]\left[\begin{array}{c}
\cos \theta \\
\sin \theta \\
1
\end{array}\right]
$$

where $\left[r 0 Z_{0} 1\right]$ is the generating point of the circle $\mathbf{C}_{3}$. Since the point $\mathbf{X}_{u}=[\cos \theta \sin \theta 1]^{\mathbf{T}}$ lies on the unit circle $\mathbf{C}_{u}=\operatorname{diag}\{1,1,-1\}$, the homography $\hat{\mathbf{H}}=\mathbf{K} \operatorname{diag}\{1,1, \gamma\}$ transforms $\mathbf{C}_{u}$ to the image of $\mathbf{C}_{3}$ as $\hat{\mathbf{C}}=\hat{\mathbf{H}}^{-\mathbf{T}} \mathbf{C}_{u} \hat{\mathbf{H}}^{-1}$, where $\gamma=Z_{0} / r$.

Now, consider the general case in which the sphere rotates about the camera center by a $3 \times 3$ rotation matrix $\mathbf{R}$. Let $\mathbf{H}=$ $\mathbf{K R} \operatorname{diag}\{1,1, \gamma\}$, the image of the sphere is then given by $\mathbf{C}=$ $\mathbf{H}^{-\mathbf{T}} \mathbf{C}_{u} \mathbf{H}^{-1}$. In the dual space, the dual of $\mathbf{C}$ is given by

$$
\begin{aligned}
\mathbf{C}^{*} & =\mathbf{K R} \operatorname{diag}\left\{1,1,-\gamma^{2}\right\} \mathbf{R}^{\mathbf{T}} \mathbf{K}^{\mathbf{T}} \\
& =\mathbf{K R}\left(\mathbf{I}+\operatorname{diag}\left\{0,0,-\left(\gamma^{2}+1\right)\right\}\right) \mathbf{R}^{\mathbf{T}} \mathbf{K}^{\mathbf{T}} \\
& =\mathbf{K} \mathbf{K}^{\mathbf{T}}-\left(\gamma^{2}+1\right) \mathbf{K} \mathbf{r}_{3} \mathbf{r}_{3}^{\mathbf{T}} \mathbf{K}^{\mathbf{T}} \\
& =\mathbf{K K}^{\mathbf{T}}-\mathbf{o o}^{\mathbf{T}},
\end{aligned}
$$

where $\mathbf{r}_{3}$ is the third column of the rotation matrix $\mathbf{R}$ and $\mathbf{o}=$ $\sqrt{\gamma^{2}+1} \mathbf{K r}_{3}$ is the image of the sphere center under $\mathbf{P}$. This result coincides with those derived in [9].

Note, due to homogenous representation, a scalar $\beta_{i}$ exists in the expression for each sphere image $\mathbf{C}_{i}$, i.e.,

$$
\beta_{i} \mathbf{C}_{i}^{*}=\omega^{*}-\mathbf{o}_{i} \mathbf{o}_{i}^{\mathbf{T}} .
$$

\section{Camera Calibration}

Based on the above derivation, this section introduces a linear approach to solve the problem of calibration.

\subsection{Calibration with Orthogonal Constraints}

By eliminating the imaged sphere centers and the scalars, the orthogonal relationship in (3) can be directly obtained for calibrating the camera.

Proposition. Given $\mathbf{C}_{1}$ and $\mathbf{C}_{2}$, which are $3 \times 3$ matrices representing two conic images, a homography $\mathbf{H}_{c}=\mathbf{C}_{2} \mathbf{C}_{1}^{*}$, termed the conic homography, can be obtained. The eigenvectors of $\mathbf{H}_{c}$ give a fixed line (axis) and a fixed point (vertex) under the transformation introduced by $\mathbf{H}_{c}$, which are also the common pole and polar with regard to $\mathbf{C}_{1}$ and $\mathbf{C}_{2}$. Specifically, if $\mathbf{C}_{1}$ and $\mathbf{C}_{2}$ are the silhouettes of two spheres, the axis and vertex become the pole and polar with regard to the image of the absolute conic.

Proof. It is straightforward to derive that the axis and vertex of $\mathbf{H}_{c}$ given by its eigenvectors, are the common pole and polar with regard to $\mathbf{C}_{1}$ and $\mathbf{C}_{2}$. Specifically, if $\mathbf{C}_{1}$ and $\mathbf{C}_{2}$ are the silhouettes of two spheres, multiplying the line $\mathbf{l}=\mathbf{o}_{1} \times \mathbf{o}_{2}$ joining the images of the two sphere centers to both sides of (6) gives

$$
\begin{aligned}
& \beta_{1} \mathbf{C}_{1}^{*} \mathbf{l}=\omega^{*} \mathbf{l} \\
& \beta_{2} \mathbf{C}_{2}^{*} \mathbf{l}=\omega^{*} \mathbf{l} .
\end{aligned}
$$

Here, $\mathbf{l}$ is also the vanishing line of the plane $\pi$ passing through the camera center and the two sphere centers. It follows that

$$
\begin{aligned}
\beta_{1} \mathbf{C}_{1}^{*} \mathbf{l}-\beta_{2} \mathbf{C}_{2}^{*} \mathbf{l} & =\omega^{*} \mathbf{l}-\omega^{*} \mathbf{l}=\mathbf{0} \\
\left(\mathbf{C}_{2} \mathbf{C}_{1}^{*}-\frac{\beta_{2}}{\beta_{1}} \mathbf{I}\right) \mathbf{l} & =\mathbf{0}
\end{aligned}
$$

Hence, $\mathbf{l}$ is an eigenvector of $\mathbf{H}_{c}$ corresponding to the eigenvalue $\beta_{2} / \beta_{1}$. l can be uniquely obtained from the eigenvectors of $\mathbf{H}_{c}$ since it is the only line having two intersection points with both conics $\mathbf{C}_{1}$ and $\mathbf{C}_{2}$.

Let $\mathbf{v}$ be the vanishing point of the normal direction of $\pi$ so that $\mathbf{l}$ and $\mathbf{v}$ satisfy the orthogonal constraint (3). From (7),

$$
\begin{aligned}
& \beta_{1} \mathbf{C}_{1}^{*} \mathbf{l}=\mathbf{v}, \\
& \beta_{2} \mathbf{C}_{2}^{*} \mathbf{l}=\mathbf{v},
\end{aligned}
$$

hence

$$
\begin{aligned}
& \frac{1}{\beta_{1}} \mathbf{C}_{1} \mathbf{v}-\frac{1}{\beta_{2}} \mathbf{C}_{2} \mathbf{v}=\mathbf{l}-\mathbf{l}=\mathbf{0}, \\
& \left(\mathbf{C}_{2}^{*} \mathbf{C}_{1}-\frac{\beta_{1}}{\beta_{2}} \mathbf{I}\right) \mathbf{v}=\mathbf{0} .
\end{aligned}
$$

This shows that $\mathbf{v}$ is an eigenvector of $\mathbf{H}_{d}=\mathbf{C}_{2}^{*} \mathbf{C}_{1}$ with corresponding eigenvalue $\beta_{1} / \beta_{2}$.

Let the eigenvectors of $\mathbf{H}_{c}$ be $\mathbf{l}_{k}$ with corresponding eigenvalues $\lambda_{k}$, i.e., $\mathbf{l}_{k}=\frac{1}{\lambda_{k}} \mathbf{C}_{2} \mathbf{C}_{1}^{*} \mathbf{l}_{k}(k=1,2,3)$. The cross product of the two eigenvectors $\mathbf{l}_{i}$ and $\mathbf{l}_{j}(i \neq j)$ is given by

$$
\begin{aligned}
\mathbf{l}_{i} \times \mathbf{l}_{j} & =\frac{1}{\lambda_{i}} \mathbf{C}_{2} \mathbf{C}_{1}^{*} \mathbf{l}_{i} \times \frac{1}{\lambda_{j}} \mathbf{C}_{2} \mathbf{C}_{1}^{*} \mathbf{l}_{j} \\
& =\frac{\operatorname{det}\left(\mathbf{C}_{2} \mathbf{C}_{1}^{*}\right)}{\lambda_{i} \lambda_{j}}\left(\mathbf{C}_{2} \mathbf{C}_{1}^{*}\right)^{-\mathbf{T}}\left(\mathbf{l}_{i} \times \mathbf{l}_{j}\right) \\
& =\frac{\lambda_{1} \lambda_{2} \lambda_{3}}{\lambda_{i} \lambda_{j}} \mathbf{C}_{2}^{*} \mathbf{C}_{1}\left(\mathbf{l}_{i} \times \mathbf{l}_{j}\right) .
\end{aligned}
$$

Without loss of generality, let $\mathbf{l}_{1}=1$, with corresponding eigenvalue $\lambda_{1}=\beta_{2} / \beta_{1}$. The cross product of the other two eigenvectors $l_{2}$ and $l_{3}$ is therefore given by

$$
\begin{gathered}
\mathbf{l}_{2} \times \mathbf{l}_{3}=\lambda_{1} \mathbf{C}_{2}^{*} \mathbf{C}_{1}\left(\mathbf{l}_{2} \times \mathbf{l}_{3}\right), \\
\left(\mathbf{C}_{2}^{*} \mathbf{C}_{1}-\frac{\beta_{1}}{\beta_{2}} \mathbf{I}\right)\left(\mathbf{l}_{2} \times \mathbf{l}_{3}\right)=\mathbf{0} .
\end{gathered}
$$

It follows that $\mathbf{v}$ is given by the cross product of the two remaining eigenvectors of $\mathbf{H}_{c}$. Hence, the axis $\mathbf{v}$ and vertex $\mathbf{l}$ of $\mathbf{H}_{c}$ are the pole and polar with regard to $\omega$. Similarly, it can also be proven that $\mathbf{l}$ is the intersection of the two remaining eigenvectors of $\mathbf{H}_{d}$ and the vertex and axis of $\mathbf{H}_{d}$ are the pole and polar with regard to $\omega$.

Note that any two spheres can be regarded as a surface of revolution (SOR), with the revolution axis given by the line passing through the two sphere centers. It is easy to see that the vertex 1 and axis $\mathbf{v}$ of the conic homography $\mathbf{H}_{c}$ correspond to the image of the revolution axis and the vanishing point of the normal direction of the plane $\pi$ passing through the camera centers and the two sphere centers, respectively. This is exploited in [3] to derive the pole-polar constraints with regard to $\omega$ from the image of a SOR.

Given two sphere images, two linear constraints on the elements of the IAC can be obtained from the axis and vertex of the conic homography $\mathbf{H}_{c}$. Hence, from three sphere images, six constraints can be obtained to fully calibrate a camera (see Fig. 2). When the number of spheres reduces to two, the camera with more than two unknown parameters cannot be calibrated. Additionally, increasing the number of spheres can increase the number of constraints and, hence, the precision of the calibration. Note that the number of 


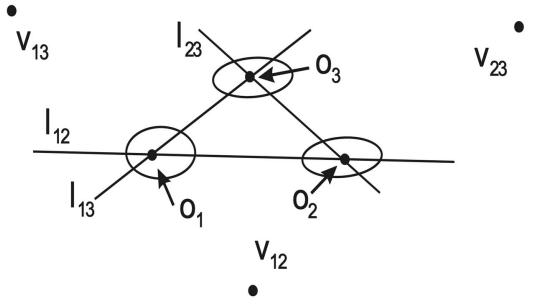

Fig. 2. Given three spheres, three conic homographies can be formed to give three pairs of axes and vertices. The camera can therefore be fully calibrated.

constraints increases nonlinearly with the number of spheres $N$ and is given by two times its combination of two, i.e., $2 \times{ }_{N} C_{2}$.

\subsection{Multicamera Calibration}

By making use of the proposed algorithm, multiple cameras can be calibrated simultaneously by imaging three or more spheres at different locations. The internal parameters $\mathbf{K}_{i}$ of each camera are first obtained and the imaged sphere centers $\hat{\mathbf{o}}_{j}^{i}$ ( $j$ being the index of the spheres) can be recovered as the intersections of the polars. The scalars $\kappa_{j}^{i}\left(\mathbf{o}_{j}^{i}=\kappa_{j}^{i} \hat{\mathbf{o}}_{j}^{i}\right)$ can therefore be easily obtained from (6). Hence, the 3D location of the sphere centers $\mathbf{O}_{j}$ with regard to the camera reference frame can be obtained as in [9], i.e.,

$$
\mathbf{O}_{j}=\mathbf{K}^{-1} \mathbf{o}_{j} .
$$

By registering the two sets of the 3D sphere centers, the camera relative rotation and translation parameters can be recovered analytically [14].

\section{EXPERIMENTS AND RESULTS}

\subsection{Synthetic Data}

The synthetic camera has focal length $f=880$, aspect ratio $\alpha=1.1$, skew $s=0.1$, and principal point $\left(u_{0}, v_{0}\right)=(320,240)$. The points on the silhouette of each sphere were corrupted with a Gaussian noise of 16 different levels from zero to three pixels, and the image of each sphere was obtained as a conic fitted to the noisy points [15].

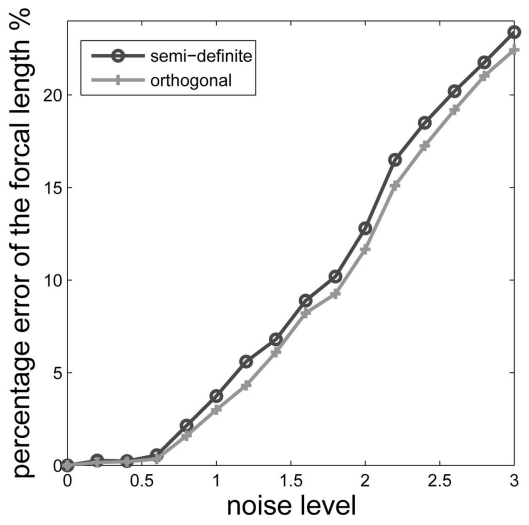

(a)
Given three sphere images, the first experiment was to calibrate the camera under different noise levels. For each level, 100 independent trials were performed using our proposed approach, as well as Agrawal's semidefinite method. Fig. 3a shows the average percentage errors of the focal length. The errors of the other parameters, which are not shown here, exhibit similar trends. It can be seen that the errors increase linearly with the noise level. From Fig. 3a, the approach with the orthogonal constraints has slightly better precision than the semidefinite approach, which may be due to fewer unknowns and calculation steps involved in the proposed approach. Table 1 shows the estimated parameters under the noise level of one pixel. The percentage errors [16] in the parameters with regard to the focal length $\alpha f$ are given in brackets.

In the second experiment, the camera was calibrated with different numbers of spheres, from three to eight, under a Gaussian noise of one pixel. For each number of spheres used, 100 independent trials were performed using the approach with orthogonal constraints, as well as Agrawal's semidefinite method. Fig. 3b shows the average percentage errors of the focal length. Due to the fast increase in the number of constraints, it can be seen that the errors decrease exponentially as the number of spheres increases. Note that the approach with orthogonal constraints again performed slightly better than the semidefinite approach.

\subsection{Real Scene}

In the real scene experiment, an image of three ping-pong balls (see Fig. 4a) was taken with a Nikon100D CCD camera. The image resolution was $1,505 \times 1,000$. The cubic B-spline snake [17] was applied to extract the apparent contours of the spheres to which conics were fitted with a least square approach [15]. The camera was calibrated with the orthogonal approach and the results were compared with those from Agrawal's semidefinite approach. The estimated parameters are listed in Table 2, where the result from the classical method of Zhang [2] is taken as the ground truth. Fig. $4 \mathrm{~b}$ shows the calibration pattern used with Zhang's calibration

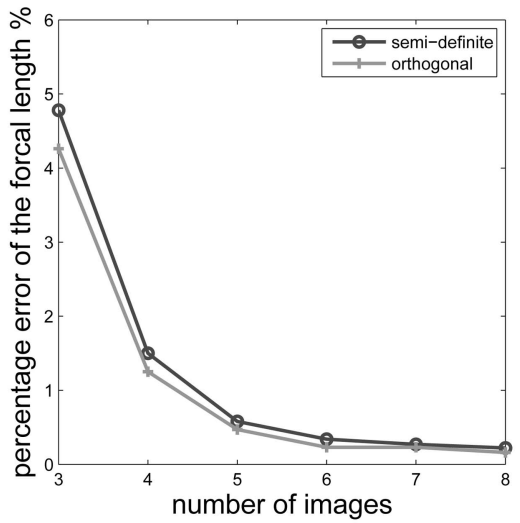

(b)

Fig. 3. (a) Relative errors of the focal length estimated under 16 different noise levels. (b) Relative errors of the focal length estimated from different numbers of imaged spheres under a noise level of one pixel.

TABLE 1

Estimated Camera Parameters from Images of Three Spheres under a Noise Level of One Pixel

\begin{tabular}{l|ccccc}
\hline Approach & $\alpha f(\%$ error $)$ & $f(\%$ error $)$ & $s(\%$ error $)$ & $u_{0}(\%$ error $)$ & $v_{0}(\%$ error $)$ \\
\hline Ground-truth & 880 & 800 & 0.1 & 320 & 240 \\
\hline Semi-definite & $838.16(4.75)$ & $766.07(3.86)$ & $2.38(0.26)$ & $334.94(1.70)$ & $238.52(0.17)$ \\
\hline Orthogonal & $839.24(4.63)$ & $769.16(3.50)$ & $1.28(0.13)$ & $324.29(0.49)$ & $242.97(0.34)$ \\
\hline
\end{tabular}




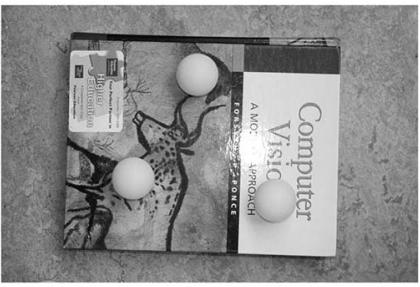

(a)

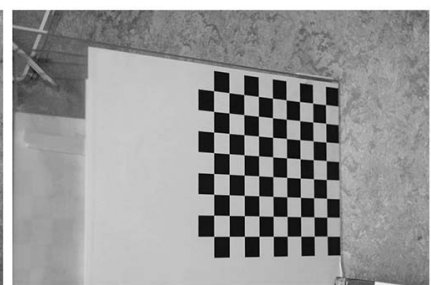

(b)
Fig. 4. (a) Image of three spheres. (b) Image of planar calibration pattern.

method. From Table 2, it can be seen that the orthogonal approach has a better performance than the semidefinite approach.

\subsection{Multicamera Calibration}

In this experiment, four spheres were imaged by a network of 15 cameras. A $9 \times 10$ grid pattern with $18 \mathrm{~mm} \times 18 \mathrm{~mm}$ squares was placed within the scene to provide background feature points for error analysis. The intrinsic parameters of each camera were first calibrated and the rotation and translation parameters of the other cameras with respect to the first one were recovered by registering the 3D sphere centers using the approach described in [14]. The fundamental matrix $\mathbf{F}_{i j}$ between an image pair was then recovered from the obtained camera intrinsic and extrinsic parameters. Note the intersection points of the pair of the inner bitangent lines to the sphere pairs provide six additional correspondences (see Fig. 5a). These points, together with the sphere centers, are mapped by $\mathbf{F}_{i j}$ to the other images. The second row of Table 3 lists the transfer errors for three arbitrary views selected from the camera network. The 72 inner corner points of the pattern in each image were extracted and mapped to the other image by the obtained $\mathbf{F}_{i j}$ and the transfer errors are listed in the third row of Table 3. Note that all the errors are about or less than one pixel. For comparison, the intrinsics of the stereo are also calibrated with Zhang's approach [2] and the transfer errors are listed in the last row of Table 3. It can be seen that the errors are smaller than those from the orthogonal constraints. This is expected as these are exactly the errors being minimized in Zhang's approach.
Note the pattern, however, will be invisible to some cameras due to back-facing, e.g., in Fig. 5b, pattern 1 is only visible to cameras 1 to 9. A second pattern was therefore put into the scene for testing the calibration results of the remaining cameras. In Fig. 5b, pattern 2 is only visible to cameras 1 and 8 to 15 . Given the recovered intrinsic and extrinsic parameters of the 15 cameras, the corner points of the two grid patterns were reconstructed and the maximum distance from any reconstructed point to its corresponding grid point is only $3.0 \mathrm{~mm}$ and $3.4 \mathrm{~mm}$ for pattern 1 and pattern 2 , respectively. Fig. $5 \mathrm{~b}$ shows the two reconstructed patterns, the four spheres, and the camera positions and orientations.

\section{Critical Configuration}

When only three spheres are being used, there are a number of critical configurations in which the calibration process fails. First, when the polar of any two sphere images passes through or is close to the principal point, the calibration will not be accurate since the corresponding pole will be at infinity. Second, when the centers of the three spheres are collinear or the plane formed by the sphere centers passes through the camera center, only two constraints can be obtained and the camera cannot be calibrated. Third, when the line joining two sphere centers passes through the camera center, the sphere limb points become concentric so that fewer constraints will be obtained. However, all of these degenerate cases can be easily avoided in practice to ensure a successful calibration, especially when more than three spheres are being used.

\section{Conclusions}

This paper has proposed a simple algorithm to calibrate a camera network by making use of the apparent contours of at least three spheres in a single image. The solution can be used as a starting point for a maximum likelihood estimation which minimizes the reprojection error of the measured edgels. The performance of calibration could be poor if the spheres are imaged near the image centers or the borders. However, the key limitation of previous approaches, namely, low precision due to error accumulation in separate steps and the introduction of extra parameters, could be alleviated using the proposed approach.

TABLE 2

Camera Parameters Estimated from the Ping-Pong Ball Image with Different Approaches

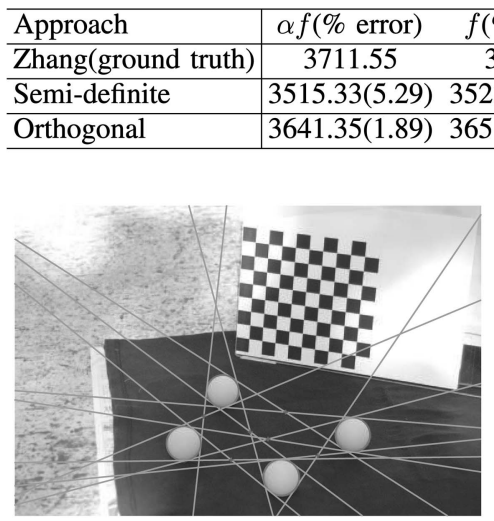

(a)

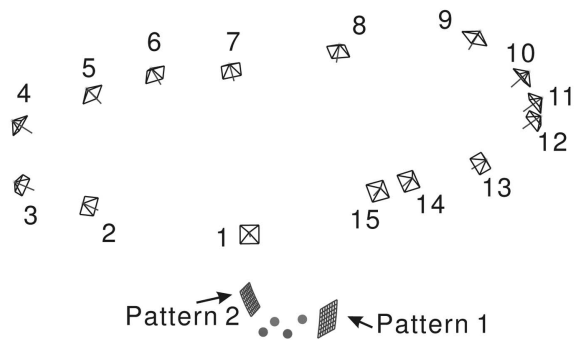

(b)

Fig. 5. (a) Image of the four spheres with a planar grid. The intersection points of the pair of the two inner bitangent lines the sphere pairs provide six correspondences. (b) The recovered grid patterns, spheres, and the camera positions and orientations. 
TABLE 3

RMS Transfer Errors (in Pixel) between Different Image Pairs in a Camera Triplet

\begin{tabular}{l|ccc}
\hline Transfer errors (pixels) & View $(1,2)$ & View(1,3) & View $(2,3)$ \\
\hline Centers \& Intersections & 0.59 & 0.87 & 0.39 \\
\hline Pattern(Orthogonal) & 0.58 & 1.03 & 0.33 \\
\hline Pattern(Zhang) & 0.45 & 0.67 & 0.53 \\
\hline
\end{tabular}

\section{REFERENCES}

[1] R.Y. Tsai, “A Versatile Camera Calibration Technique for High Accuracy 3D Machine Vision Metrology Using Off-the-Shelf TV Cameras and Lenses," IEEE J. Robotics and Automation, vol. 3, no. 14, pp. 323-344, 1987.

[2] Z. Zhang, "A Flexible New Technique for Camera Calibration," IEEE Trans. Pattern Analysis and Machine Intelligence, vol. 22, no. 11, pp. 1330-1334, Nov. 2000.

[3] K.-Y.K. Wong, P.R.S. Mendonça, and R. Cipolla, "Camera Calibration from Surfaces of Revolution," IEEE Trans. Pattern Analysis and Machine Intelligence, vol. 25, no. 2, pp. 147-161, Feb. 2003

[4] H. Teramoto and G. Xu, "Camera Calibration by a Single Image of Balls: From Conics to the Absolute Conic," Proc. Fifth Asian Conf. Computer Vision, pp. 499-506, 2002.

[5] X. Ying and Z. Hu, "Catadioptric Camera Calibration Using Geometric Invariants," IEEE Trans. Pattern Analysis and Machine Intelligence, vol. 26, no. 10 , pp. $1260-1271$, Oct. 2004

[6] P. Beardsley, D. Murray, and A. Zisserman, "Camera Calibration Using Multiple Images," Proc. European Conf. Computer Vision, pp. 312-320, 1992.

[7] M.A. Penna, "Camera Calibration: A Quick and Easy Way to Determine the Scale Factor," IEEE Trans. Pattern Analysis and Machine Intelligence, vol. 13, no. 12, pp. 1240-1245, Dec. 1991.

[8] D. Daucher, M. Dhome, and J. Lapreste, "Camera Calibration from Spheres Images," Proc. European Conf. Computer Vision, pp. 449-454, 1994.

[9] M. Agrawal and L.S. Davis, "Camera Calibration Using Spheres: A SemiDefinite Programming Approach," Proc. IEEE Int'l Conf. Computer Vision, pp. 782-789, 2003

[10] H. Zhang, G. Zhang, and K.-Y.K. Wong, "Camera Calibration with Spheres: Linear Approaches," Proc. Int'l Conf. Image Processing, vol. II, pp. 1150-1153, Sept. 2005.

[11] R.I. Hartley and A. Zisserman, Multiple View Geometry in Computer Vision. Cambridge Univ. Press, 2000.

[12] O.D. Faugeras, Q.-T. Luong, and S.J. Maybank, "Camera Self-Calibration: Theory and Experiments," Proc. European Conf. Computer Vision, pp. 321334, 1992.

[13] J.E. Gentle, Numerical Linear Algebra for Applications in Statistics. SpringerVerlag, 1998.

[14] P. Besl and N. McKay, "A Method for Registration of 3D Shapes," IEEE Trans. Pattern Analysis and Machine Intelligence, vol. 14, no. 2, pp. 239-256, Feb. 1992.

[15] A.W. Fitzgibbon, M. Pilu, and R.B. Fisher, “Direct Least-Squares Fitting of Ellipses," IEEE Trans. Pattern Analysis and Machine Intelligence, vol. 21, no. 5, pp. 476-480, May 1999.

[16] Z. Zhang, "Camera Calibration with One-Dimensional Objects," IEEE Trans. Pattern Analysis and Machine Intelligence, vol. 26, no. 7, pp. 892-899, July 2004.

[17] R. Cipolla and A. Blake, "Surface Shape from the Deformation of Apparent Contours," Int'l J. Computer Vision, vol. 9, no. 2, pp. 83-112, Nov. 1992. 